\title{
CONTEXTO FAMILIAR E O DESEMPENHO DE ESTUDANTES DO 5 ANO DE UMA ESCOLA NO INTERIOR DE SÃO PAULO
}

\author{
THE FAMILIAR CONTEXT AND THE PERFORMANCE OF $5^{\text {th }}$ GRADE STUDENTS FROM A \\ SCHOOL IN THE INNER REGION OF SÃO PAULO
}

El CONTEXTO FAMILIAR Y EL APROVECHAMIENTO DE LOS ESTUDIANTES DEL 5 AÑO DE UNA ESCUELA DEL INTERIOR DE SÃO PAULO

Andreia Osti ${ }^{1}$

\begin{abstract}
RESUMO
O presente artigo analisa a relação entre o ambiente familiar, considerando seus recursos materiais e humanos e o desempenho escolar de alunos do ensino fundamental. A amostra foi composta por 120 alunos do $5^{\circ}$ ano da rede pública municipal da Região Metropolitana de Campinas, sendo 60 estudantes com satisfatório desempenho escolar e 60 com desempenho insatisfatório, segundo a avaliação dos professores. O instrumento utilizado para avaliar os recursos humanos e materiais foi um questionário sobre os suportes e recursos do ambiente familiar elaborado a partir do Inventário de Recursos do Ambiente Familiar (RAF), aplicado coletivamente. Os resultados indicam que há diferenças entre os grupos em relação aos recursos humanos e culturais, bem como na interação familiar e participação na escola. De maneira geral, pode-se afirmar que crianças com satisfatório desempenho escolar relatam terem tido maior acesso a atividades extracurriculares e maiores oportunidades de interação com seus pais. Também afirmam existir no cotidiano da casa uma rotina pré-estabelecida para a realização das atividades do dia. O grupo com desempenho insatisfatório, em relação a todas as categorias descritas, apresentou menores índices. Esse trabalho contribui para o entendimento da importância da interação familiar para o desempenho escolar, refletida nas possibilidades de diálogo, de compartilhar uma atividade doméstica e ao acompanhar o processo de aprendizagem da criança.
\end{abstract}

PALAVRAS-CHAVE: Família. Rendimento escolar. Aprendizagem.

\begin{abstract}
This article analyzes the relationship between the family ambience, considering its material and human resources and school performance of primary school students. The sample consisted of 120 students from the $5^{\text {th }}$ grade of a municipal school in the Metropolitan Region of Campinas, 60 of those students having satisfactory academic performance and 60 unsatisfactory performance, according to their teacher's evaluation. The instrument used to evaluate the human and material resources was a questionnaire about the backups and resources from the familiar ambience elaborated from the Resource Inventory of Home Ambience (RAF), applied collectively. The results indicate that there are differences between the groups in relation to human and cultural resources, as well as in family interaction and participation in the school. Overall, it can be affirmed that children with satisfactory school performance report having had greater access to extracurricular activities and greater opportunities of interaction with their parents. They also affirm having a preestablished routine in the house to accomplish the school activities. The group with unsatisfactory performance, in relation to all the categories described, presented lower rates. This work contributes to the understanding of the importance of family interaction for school performance, reflected in the possibilities of dialogue, to sharing a household activity and monitoring the child's learning process.
\end{abstract}

KEYWORDS: Family. School Performance. Learning.

\footnotetext{
${ }^{1}$ Doutora em Educação pela Universidade Estadual de Campinas, UNICAMP, Campinas, SP - Brasil. Professora do Departamento de Educação do Instituto de Biociências de Rio Claro, Universidade Estadual Paulista "Júlio de Mesquita Filho", UNESP, Rio Claro, SP - Brasil. E-mail: andreia.osti@gmail.com.

Recebido em: 30/06/2015 - Aprovado em: 13/06/2016.
} 


\section{RESUMEN}

El presente artículo analiza la relación entre el ambiente familiar, considerando sus recursos materiales y humanos, y el aprovechamiento escolar de alumnos de la enseñanza primaria. La muestra se realizó con 120 alumnos del $5^{\circ}$ año de la red pública municipal de la Región Metropolitana de Campinas, siendo 60 estudiantes con aprovechamiento escolar satisfactorio y 60 con aprovechamiento insatisfactorio, según la evaluación de los profesores. El instrumento utilizado para evaluar los recursos humanos y materiales fue un cuestionario sobre los soportes y recursos del ambiente familiar elaborado a partir del Inventario de Recursos del Ambiente Familiar (RAF), aplicado colectivamente. Los resultados indican que hay diferencias entre los grupos con relación a los recursos humanos y culturales, así como en la interacción familiar y la participación en la escuela. De manera general, se puede afirmar que los niños con aprovechamiento escolar satisfactorio relatan haber tenido mayor acceso a actividades extracurriculares y mayores oportunidades de interacción con sus padres. También afirman que existe en el cotidiano de la casa una rutina preestablecida para la realización de las actividades del día. El grupo con aprovechamiento insatisfactorio, en relación a todas las categorías descritas, presentó índices menores. Este trabajo contribuye al entendimiento de la importancia de la interacción familiar para el aprovechamiento escolar, reflejada en las posibilidades de diálogo, de compartir una actividad doméstica y de acompañar el proceso de aprendizaje del niño.

PALABRAS CLAVE: Familia. Aprovechamiento escolar. Aprendizaje.

\section{INTRODUÇÃO}

A família e o contexto social são influências para a criança e o adolescente no que diz respeito ao seu desenvolvimento emocional, social e para sua aprendizagem. (Guidetti \& Martinelli, 2009). No que se refere à aprendizagem, objeto de atenção do presente estudo, as crianças que tenham apoio familiar, estímulo e supervisão em suas tarefas escolares de forma mais efetiva e constante, tendem a apresentar melhor desempenho acadêmico, quando comparadas às crianças que não possuam supervisão mais direta em seus estudos. Quando se analisa o desempenho escolar é impossível dissociá-lo do contexto em que o aluno vive, incluindo o âmbito familiar e a escola. Nesse sentido, o núcleo familiar é revestido de importância capital, pois é o espaço que oferece uma infinidade de estímulos para o desenvolvimento infantil, além de ser o primeiro ambiente psicossocial, protótipo das relações a serem estabelecidas com o mundo (Engleend \& Luckner, 2004, Randall, 2005, Patrinos, 2006, Silva e Varani, 2009).

Pesquisas nacionais (Carvalho, 2004, Rueda \& Bartholomeu, 2006, Guidetti, 2007, Aguena, 2010, Osti, 2010, Osti e Brenelli, 2011, Santos, Martinelli \& Monteiro, 2012, Osti \& Martinelli, 2014) e internacionais (Engleend \& Luckner, 2004, Randall, 2005, Patrinos, 2006, Steensel, 2006) evidenciam a importância de conhecer a influencia da família nas diferentes fases e nos aspectos do desenvolvimento da criança. Em comum, os autores consideram que o suporte parental pode ser determinante para o sucesso ou fracasso de alunos na medida em que necessitam no decorrer de seu processo de escolarização, de apoio e orientação para enfrentar as diversas situações vivenciadas na escola.

Ao investigar a associação entre a qualidade do estímulo doméstico e o desempenho cognitivo infantil, Andrade (2005) destaca que quanto melhor a qualidade da estimulação ambiental disponível para a criança, melhor o seu desempenho cognitivo. Em consonância, Carvalho (2004) afirma que os pais que conseguem dispor de tempo mínimo para dar atenção a seus filhos e mostrar que se importam com seus deveres escolares e com sua aprendizagem, podem favorecer a vida escolar deles, tornando-os mais disciplinados e empenhados a resolver as tarefas que lhe cabem, 
tendo assim mais chances de sucesso escolar. Tal como preconizado por Cooper, Lindsay e Nye (1999) há mais de uma década, a família tem papel chave no sucesso escolar de crianças e os pais podem contribuir para o desenvolvimento intelectual de seus filhos de diversas formas: preparando as crianças para a escola, valorizando e incentivando a educação, estabelecendo hábitos de estudo, ajudando com os deveres, acompanhando o progresso acadêmico e fornecendo apoio em todas as atividades escolares.

Jiang (2004) encontrou vários indicadores associados com os efeitos do ambiente familiar no desempenho escolar, entre eles a ocupação dos pais, instrução e renda, recursos da família e participação parental na escola. Hoje, a família é interpretada como um contexto complexo promotor do desenvolvimento primário, da sobrevivência e da socialização da criança, e também um espaço de transmissão de cultura, significado social e conhecimento comum agregado ao longo das gerações (DESSEN; BRAZ, 2005). Para além dessas funções também é responsabilidade da família acompanhar e dar suporte ao desenvolvimento escolar de seus filhos.

D’Avila, Bacarji, Marturano e Elias (2005) destacam três formas de suporte que a família pode proporcionar à criança: suporte específico para a realização escolar, suporte ao desenvolvimento e suporte emocional. O primeiro concretiza-se pelo envolvimento dos pais na vida escolar dos filhos, o que implica em disposição de tempo e espaço para a realização das tarefas escolares, bem como o estabelecimento de uma rotina doméstica. $\mathrm{O}$ segundo reflete, dentre outros quesitos, na priorização de atividades de lazer entre pais e filhos. Por fim, o suporte emocional diz respeito ao clima emocional e às relações afetivas da família, o que implica nas interações familiares. Segundo Santos, Martinelli e Monteiro (2012) o equilíbrio entre esses três aspectos favorece o desenvolvimento cognitivo e o desempenho escolar, gerando maior propensão para o ajustamento interpessoal da criança.

Ao verificar as associações entre problemas de comportamento de crianças e variáveis do ambiente familiar, Ferreira e Marturano (2002) identificaram que crianças sem problemas de comportamento (indisciplina, agressividade verbal e física), têm maior supervisão dos pais em relação à organização do tempo e do espaço para a realização de tarefas escolares, dispõem de mais materiais que auxiliam na aprendizagem, realizam passeios e viagens com os pais, além de interagir verbalmente e compartilhar histórias em casa. Com propósito semelhante, D'Avila, Bacarji, Marturano e Elias, (2005) compararam o suporte parental oferecido às crianças com queixa de dificuldade de aprendizagem e crianças sem dificuldades. Os resultados indicam que crianças com dificuldades apresentam um clima afetivo no ambiente familiar menos favorável, o que sugere que a relação conflituosa entre pais e filhos pode afetar o desempenho escolar.

Ao analisar as percepções de adolescentes sobre os suportes parentais, Spera (2006) encontrou relação significativa entre os suportes oferecidos pelos pais e o desempenho dos filhos. Investigando a relação entre a participação familiar e as atividades escolares, Carvalho (2012) observou que os alunos que habitualmente fazem o dever de casa contam com a ajuda dos pais e esses se mostram interessados. Em contrapartida, os alunos que não costumam fazer o dever de casa, não podem contar com ajuda dos pais e esses são mais ausentes. Para esse autor, contar com o 
auxílio dos pais no dever de casa pode melhorar a qualidade da aprendizagem dos alunos. Mais recentemente, Barbosa (2011) afirma que a educação dos pais e o contexto familiar apresentam-se como fatores relevantes para a trajetória escolar dos estudantes.

Em síntese, a literatura vinculada aos recursos do ambiente familiar tem evidenciado que a família é o componente influenciador mais crítico das habilidades das crianças para o desempenho na escola (Miller, 1999) e que o contexto sociocultural da criança pode possibilitar o aperfeiçoamento de suas potencialidades, favorecendo seu enriquecimento pessoal (Madaus, Airasian, Kellaghan, 2008, Guidetti \& Martinelli, 2009, Barbosa, 2011). Outras pesquisas apontam que as expectativas parentais têm efeito direto no desempenho escolar das crianças (Engleend \&Luckner, 2004) assim como afirmam que as práticas familiares podem explicar o diferencial das crianças na escola (Cooper, Lindsay e Nye, 1999, Marchesi, 2006).

Estudos internacionais (Lindsay \& Nye, 1999, Bradley \& Corwyn, 2002; Cooper, Engleend \& Luckner, 2004; Jiang, 2004; Spera, 2006, Madaus, Airasian \& Kellaghan, 2008) também mostram que uma série de condições presentes no ambiente familiar são fortes preditores do desempenho escolar. Entre essas condições, pode-se destacar o clima emocional familiar positivo, o incentivo parental às atividades escolares, a valorização da educação, o estabelecimento de hábitos de estudo e o acompanhamento escolar. Sabe-se que a família participa de maneira efetiva na formação da criança e que o suporte ao seu desenvolvimento reflete uma disposição dos pais para investir tempo e recursos em arranjos da vida familiar.

Em relação à postura dos pais em prestar toda a assistência que a criança necessite e incentivar sua independência, bem como estabelecer rotinas cotidianas e regras, Marturano (2006) considera que essas ações constituem aspectos importantes que facilitam a discriminação, por parte da criança, dos processos de controle vigentes dentro da escola. Assumindo essa assertiva e acreditando que o aprender deve ser pensado como uma responsabilidade compartilhada, uma vez que envolve professores, alunos e família, esse trabalho concebe que a análise dos recursos humanos e materiais da família constituem importante aspecto a ser analisado junto ao desempenho escolar.

Cabe esclarecer que nesse trabalho os recursos estão sendo entendidos da seguinte forma: os recursos humanos são aqueles inerentes à vida em família, ou seja, a oportunidade de interação e diálogo entre pais e filhos, o que compreende desde compartilhar atividades no lar, tal como fazer uma refeição juntos ou executar uma tarefa doméstica, estabelecer uma organização das rotinas, até realizar uma viagem ou passeio. Os recursos materiais compreendem atividades programadas que a crianças realizam regularmente e a oferta de materiais promotores de desenvolvimento (livros, jornais, brinquedos, dentre outros). Partimos da premissa que o desempenho escolar implica uma reunião de fatores que incluem desde os recursos materiais e humanos até a qualidade da relação entre aluno e professor, a participação efetiva da família junto à escolarização da criança, o compromisso da escola, a adaptação, e cumprimento dos deveres escolares por parte do aluno, dentre outros. No entanto, nessa pesquisa, priorizou-se analisar o desempenho em relação aos recursos do ambiente familiar. 
Considerando que é importante determinar os recursos do ambiente familiar que favorecem o desempenho escolar, Marturano (1999) elaborou o Inventário de Recursos do Ambiente Familiar (RAF) que permite analisar informações sobre condições relevantes para o desempenho acadêmico. Uma revisão de pesquisas que utilizaram o RAF (Santos e Marturano, 1999, D'Avila, Bacarji, Marturano, Elias, 2004, Trivellato-Ferreira, 2005, Guidetti, 2007) indica que o inventário é uma ferramenta útil para pesquisadores que trabalham com o contexto educacional, uma vez que permite rastrear informações e implementar práticas de orientação pedagógica às famílias.

A justificativa desta pesquisa se apóia no fato de que a influência do ambiente familiar deve ser encarada como importante fator frente ao desempenho acadêmico. Justifica-se também a necessidade de esclarecimento, em relação aos professores e demais profissionais da educação, sobre a influência do ambiente familiar no desempenho de alunos, uma vez que investigações (Caetano, 2003; Engleend \& Luckner, 2004; Guidetti, 2007; Madaus, Airasian \& Kellaghan, 2008, Dessen, 2011) têm revelado que é imprescindível que o aluno tenha acompanhamento familiar para conseguir aprender e que, dentre os fatores considerados importantes para o sucesso na aprendizagem, destacase a participação dos pais na vida escolar de seus filhos.

No presente artigo buscou-se compreender se os recursos materiais e humanos do ambiente familiar podem contribuir para o sucesso ou insucesso acadêmico de alunos que tem ou não apoio escolar em casa. Consideramos que o estudo sobre o tema é atual para a área educacional, uma vez que poderá contribuir para salientar a importância da aliança e parceira entre família e escola na e para a melhoria da qualidade de aprendizagem de crianças e adolescentes.

\section{MÉTODO}

\section{Configuração do ambiente escolar}

A escola em que a pesquisa foi realizada é uma escola pública da rede municipal de uma cidade no interior de São Paulo, localizada na região metropolitana de Campinas, selecionada em decorrência de apresentar altos índices de alunos que apresentam dificuldades para aprender. A unidade escolar atende alunos do primeiro ao nono ano do ensino fundamental e funciona no período matutino e vespertino. A unidade escolar apresenta como característica peculiar o fato dos alunos serem residentes do mesmo bairro em que ela se localiza, havendo uma proximidade física entre o prédio escolar e as casas dos estudantes. Destaca-se que não foi foco de análise a situação econômica e a escolaridade dos pais.

\section{Participantes}

Os participantes deste estudo foram 120 alunos de cinco salas de uma mesma escola da rede pública municipal da Região Metropolitana de Campinas, sendo 51 (42,5\%) do sexo feminino e 69 $(57,5 \%)$ do masculino, com idades entre 10 e 14 anos. Todos os estudantes cursavam o $5^{\circ}$ ano do Ensino Fundamental e foram indicados por seus professores como sendo representantes de satisfatório e insatisfatório desempenho. A configuração dos grupos foi feita pelos docentes baseada nas notas dos estudantes em relação ao ano anterior e atual, e em seu desempenho geral em sala de 
aula. As instruções foram dadas aos professores coletivamente em três encontros durante a hora de trabalho pedagógico na escola. Do total de participantes, 60 foram classificados por seus professores como possuindo satisfatório desempenho e $60 \mathrm{com}$ desempenho insatisfatório. Cabe esclarecer que os alunos com desempenho insatisfatório foram considerados por seus professores como aqueles que apresentam dificuldade para aprender, atraso em relação aos conteúdos ensinados e notas abaixo da média mínima esperada. Destaca-se que a participação dos professores foi apenas no sentido de sugerir a organização dos dois grupos de alunos. Os cinco professores das cinco salas investigadas participaram fazendo a indicação dos estudantes. Todos os professores são efetivos na escola, regentes das salas em que os alunos estudavam e com o mínimo de seis anos de trabalho nessa mesma escola.

\section{Instrumentos}

Foi aplicado um questionário sobre os suportes e recursos do ambiente familiar (Guidetti e Martinelli, 2005) elaborado a partir do Inventário de Recursos do Ambiente Familiar (RAF) de Marturano (1999). O questionário avalia como a criança identifica o suporte familiar oferecido a ela e os recursos disponíveis no seu ambiente familiar. O instrumento é constituído por oito tópicos utilizando palavras que fazem parte do repertório infantil e em cada um deles há aproximadamente oito alternativas para a criança assinalar qual (quais) condiz (em) com sua realidade. Os resultados são analisados mediante a soma das médias obtidas nos tópicos, dividida pelo número de itens que o compõem, multiplicado por 10. O escore total corresponde a soma dos escores obtidos nos oito tópicos que estão reunidos em três categorias: supervisão parental das rotinas escolares, interação com os pais e recursos do ambiente físico.

\section{Procedimento de coleta dos dados}

O estudo foi iniciado no primeiro semestre de 2014 após o consentimento dos pais e/ou responsáveis legais dos alunos, de acordo com o definido na Resolução CNS 466/2012, e após trazerem o Termo de Consentimento Livre e Esclarecido assinado em duas vias. Foram consultados 150 pais e desses, 138 autorizaram a participação de seus filhos, entretanto no dia da coleta 18 estudantes faltaram. Somente integraram a pesquisa as crianças cujos pais autorizaram a participação. O instrumento foi aplicado coletivamente na própria escola, durante o horário da aula da disciplina de Língua Portuguesa em meados de junho, quando os professores já haviam fechado as notas do primeiro semestre letivo. A aplicação durou em média 20 minutos e ocorreu em um mesmo dia para todos os alunos. A professora da sala fez a leitura do instrumento, na presença da pesquisadora que permaneceu, durante toda a coleta, dentro da sala. A leitura do instrumento foi feita em voz alta, sendo acompanhada pelos estudantes, que foram assinalando os itens que correspondiam a sua realidade. Cabe esclarecer que todos os tópicos foram respondidos. 


\section{RESULTADOS E DISCUSSÃO}

Com o objetivo de melhor compreender a relação entre o desempenho e os recursos humanos e materiais e para facilitar a análise, os dados do inventário foram subdivididos e organizados em quatro categorias: 1) recursos do ambiente físico, 2) recursos culturais, 3) dinâmica e interação familiar e 4) participação familiar em relação à escola. A Tabela 1 informa sobre as estatísticas descritivas.

TABELA 1- Recursos do ambiente físico

\begin{tabular}{lcccc}
\hline Categorias & Desempenho Satisfatório & Desempenho Insatisfatório \\
& $\mathrm{N}$ & $\%$ & $\mathrm{~N}$ & $\%$ \\
Brincar dentro de casa & 47 & 78,3 & 17 & 28,3 \\
Brincar na rua & 13 & 21,6 & 43 & 71,6 \\
Variedade de brinquedos & 49 & 81,6 & 36 & 60 \\
Acervo geral de livros & 57 & 95 & 54 & 90 \\
Acervo de livros escolares & 16 & 26,6 & 39 & 65 \\
Acervo de livros de diferentes gêneros & 41 & 58,3 & 15 & 25 \\
Total & 60 & 100 & 60 & 100 \\
\hline
\end{tabular}

Fonte: Elaborada pela autora.

No que se refere aos recursos do ambiente físico, os dados permitem afirmar que há diferenças entre os grupos. Crianças com desempenho satisfatório brincam dentro de casa (78,3\%) e possuem um maior acervo e variedade de tipos de brinquedo (81,6\%), tais como: instrumento musical, jogos de números, de letras e palavras, de classificação de objetos, de regras, dentre outros. Os alunos com desempenho insatisfatório brincam principalmente na rua $(71,6 \%)$, ou seja, fora de casa, embora em relação ao acervo de livros, ambos os grupos o possuem (95\% desempenho satisfatório e $90 \%$ desempenho insatisfatório). No entanto, alunos sem dificuldades possuem em maior proporção $(58,3 \%)$, livros de diferentes gêneros, tais como de literatura, infantis, técnicos, jornais e revistas de notícias. O grupo com dificuldade possui livros relacionados aos conteúdos escolares $(65 \%)$, como dicionário e livros didáticos.

Os dados corroboram a literatura (Marturano, 1998, Carvalho, 2000, D'avila, Bacarji, Marturano, \& Elias, 2005, Guidetti, 2007, Barbosa 2011), ao indicarem que quanto maiores os recursos do ambiente familiar, maiores as diferenças no desempenho escolar infantil. Nessa mesma direção, Jiang (2004) afirma que os recursos do ambiente familiar estão diretamente associados com o desempenho educacional dos estudantes. A tabela 2 apresenta os dados em relação aos recursos culturais. Cabe esclarecer que os recursos culturais aqui são entendidos como oportunidades proporcionadas pela família à criança em relação a frequentar exposições, teatros, cinemas, manifestações artísticas, festas, passeios, dentre outros. Ou seja, configuram situações em que essa criança possa ter contato e/ou conhecer diferentes ambientes, bem como ter acesso a diferentes contextos culturais. 
TABELA 2 - Recursos culturais

\begin{tabular}{lcccc}
\hline \multicolumn{1}{c}{ Categorias } & \multicolumn{2}{c}{ Desempenho } & \multicolumn{2}{c}{$\begin{array}{c}\text { Desempenho } \\
\text { Insatisfatório }\end{array}$} \\
& \multicolumn{2}{c}{ Satisfatório } & $\mathrm{N}$ & $\%$ \\
Passeios no centro da cidade & $\mathrm{N}$ & $\%$ & 60 & 100 \\
Passeios diferenciados & 60 & 100 & 04 & 6,6 \\
Viajem de avião & 43 & 71,6 & 0 & 0 \\
Curso extracurricular & 03 & 5 & 17 & 28,3 \\
Total & 52 & 86,6 & 60 & 100 \\
\hline
\end{tabular}

Fonte: Elaborada pela autora.

Em relação aos resultados desta pesquisa, ambos os grupos realizam passeios ao centro da cidade, tal como ir ao shopping, lanchonetes e passear na praça ou centro comercial. Entretanto, verifica-se que os indivíduos com desempenho satisfatório, realizaram mais passeios específicos e em diferentes lugares nos últimos doze meses $(71,6 \%)$ tal como: bosque, circo, cinema ou teatro, parque de diversões, praia, exposições, tendo viajado inclusive de avião (5\%). Esse grupo também tem maior acesso a atividades extracurriculares como curso de computação e inglês $(86,6 \%)$. Em outra direção, os alunos com desempenho insatisfatório, nos últimos doze meses, praticamente não realizaram passeios fora da cidade $(6,66 \%)$, e em menor proporção frequentaram cursos extracurriculares $(28,3)$.

Nesse sentido, há de se destacar o ambiente familiar como um dos fatores que contribui para o desempenho, na medida em que os adultos que tem tempo e disposição para interagir com a criança atuam como mediadores entre escola e família. No entanto, adicionalmente, algumas pesquisas enfatizam que quanto maior a presença de recursos no ambiente, maior probabilidade de aproveitamento escolar (Jiang, 2004; Midgett, Belsito, Ryan \& Adam, 2000, Swarttz, 2003,) e que quanto menor o desempenho acadêmico, menos recursos no ambiente familiar (Guidetti, 2007, Barbosa, 2011). A Tabela 3 descreve os dados em relação à dinâmica e interação familiar.

TABELA 3- Dinâmica familiar

Categorias

Conversar como foi o dia Conversar sobre notícias da TV Atividades domésticas em parceria Família reunida à noite

Família reunida nos finais de semana

Familiar reunida em passeios

Rotina de horários definida

Total

\begin{tabular}{ll}
\multicolumn{2}{l}{ Desempenho } \\
Satisfatório \\
$\mathrm{N}$ & $\%$ \\
49 & 81 \\
42 & 70 \\
57 & 95 \\
25 & 41,6 \\
41 & 68,3 \\
43 & 71,6 \\
44 & 73,3 \\
60 & 100
\end{tabular}

\begin{tabular}{lc}
\multicolumn{2}{l}{ Desempenho } \\
Insatisfatório \\
$\mathrm{N}$ & $\%$ \\
33 & 55 \\
19 & 31,6 \\
37 & 61,6 \\
17 & 28,3 \\
22 & 36,6 \\
05 & 8,3 \\
20 & 33,3 \\
60 & 100
\end{tabular}

Fonte: Elaborada pela autora.

Ao analisar a dinâmica familiar, pode-se observar que nesse grupo os alunos com desempenho satisfatório têm maiores oportunidades de interação com seus pais, uma vez que a família costuma estar reunida durante a semana no período da noite tanto para jantar quanto para assistir televisão (41,6\%), compartilham informações sobre a rotina do dia (81\%) e seus membros permanecem juntos aos finais de semana realizando atividades em comum (68,3\%). Também realizam atividades domésticas como lavar a louça, arrumar a casa e fazer almoço em parceria 
(95\%). No cotidiano da casa existe uma rotina estabelecida $(73,3 \%)$ em que as crianças têm hora para brincar, fazer a lição, ver televisão, tomar banho e ir dormir. Adicionalmente, pais e filhos têm mais diálogo sobre como foi o dia na escola, comentam a respeito de programas na TV, contam histórias, bem como conversam sobre assuntos diversos, o que pode configurar um fator de diferença no desempenho (Carvalho, 2012; Jiang, 2004; Miller, 1999).

Os dados permitem inferir que a interação dos pais com os filhos e o contexto familiar podem ser fontes de recursos para que a criança tenha um melhor aproveitamento escolar e apresente desenvolvimento sadio, aprendendo a lidar com suas dificuldades. Em outra direção, pode levar a reações de inadaptação e influenciar seu desempenho escolar negativamente. A Tabela 4 informa sobre a participação familiar na escola.

TABELA 4 - Envolvimento familiar na escolarização

\begin{tabular}{llllc}
\hline \multicolumn{1}{c}{ Categorias } & \multicolumn{2}{c}{ Desempenho } & \multicolumn{2}{c}{ Desempenho } \\
& \multicolumn{2}{c}{ Satisfatório } & \multicolumn{2}{c}{ Insatisfatório } \\
& $\mathrm{N}$ & $\%$ & $\mathrm{~N}$ & 11,6 \\
Verificar o material escolar & 35 & 58,3 & 07 & 18,3 \\
Supervisionar a lição de casa & 50 & 83,3 & 11 & 15 \\
Auxiliar no estudo para as provas & 38 & 63,3 & 09 & 40 \\
Comparecer as reuniões escolares & 54 & 90 & 24 & 38,3 \\
Acompanhar as notas e frequências & 55 & 91,6 & 23 & 100 \\
Total & & & 60 & \\
\hline
\end{tabular}

Fonte: Elaborada pela autora.

A participação familiar tem sido entendida aqui, como a presença constante dos pais em atividades da educação escolar de seus filhos. Os resultados permitem afirmar que os alunos com desempenho satisfatório tiveram sistematicamente percentagens maiores quando comparados aos alunos com desempenho insatisfatório. Mais particularmente, os alunos com desempenho satisfatório contam com a participação dos pais na supervisão da lição de casa $(83,3 \%)$, na orientação para o estudo de provas $(63,3 \%)$ e na verificação do material escolar $(58,3 \%)$. Os pais desse grupo, com frequência maior que o outro grupo, comparecem as reuniões escolares (90\%), acompanham as notas e a frequência escolar de seus filhos $(91,6 \%)$. O grupo de alunos com desempenho insatisfatório também contam, mas em menor percentagem, com a participação dos pais na supervisão de atividades escolares $(18,3 \%)$. Há maior índice de ausência da participação dos pais nas reuniões escolares $(60 \%)$ e na supervisão das tarefas $(81,7 \%)$.

Considera-se que o envolvimento dos pais em atividades escolares possibilita ao filho uma melhor assistência ao seu desenvolvimento, bem como o encorajamento e a valorização por sua escolarização. Pode-se inferir, mediante os dados, que os alunos com desempenho insatisfatório têm menos recursos materiais e humanos, tal como previsto teoricamente. Segundo D'avila, Bacarji, Marturano e Elias (2005), Guidetti (2007) e Barbosa (2011), as variáveis da organização familiar podem explicar a maior parte da variação entre o desempenho dos alunos brasileiros. Também afirmam que ter um lugar calmo para estudar, acesso ao computador e fazer a lição de casa são fatores que provocam melhora no desempenho.

Embora a posição social dos alunos não tenha sido considerada em nossa análise, esclarecemos que há evidências de que a disponibilidade de recursos, a oportunidade de vivenciar diferentes experiências culturais e as disposições familiares para a educação dos filhos não são igualmente distribuídas na sociedade, mesmo dentro do microcosmo de uma escola pública situada 
na periferia em que a totalidade dos alunos estuda e reside no mesmo bairro e cujos pais são igualmente trabalhadores de baixa renda. No entanto, mesmo buscando uma similaridade de condições, nessa pesquisa, encontramos diferenças entre o desempenho dos estudantes e os recursos do contexto familiar. Esses dados nos permitem afirmar que um dos fatores que podem vir a impactar o desempenho dos alunos é o contexto familiar, ou seja, a efetiva participação da família na escolarização de seus filhos.

\section{CONSIDERAÇÕES FINAIS}

A presente pesquisa investigou a relação entre o desempenho escolar de alunos do ensino fundamental com desempenho satisfatório e insatisfatório e os recursos materiais e humanos do ambiente familiar. Para isso foram definidas quatro categorias de análise, são elas: os recursos do ambiente físico, o recurso cultural, a dinâmica e interação familiar e a participação familiar em relação à escola.

De maneira geral, pode-se afirmar que o grupo com desempenho escolar satisfatório possui mais recursos materiais e culturais, o que pode ser verificado, desde a quantidade e variedade de brinquedos e livros de diferentes gêneros, passeios realizados no último ano junto com a família, bem como a participação em aulas extracurriculares. Também tem maiores oportunidades de diálogo e interação em diferentes atividades com seus pais. As famílias desse grupo estabelecem uma rotina doméstica em que os filhos têm horários definidos em casa para a realização de atividades. Em relação à participação familiar na educação dos filhos, o grupo com desempenho satisfatório apresenta maior frequência nas reuniões escolares, bem como na supervisão e orientação das atividades escolares.

Os dados sugerem que o grupo com desempenho insatisfatório dispõe de menores recursos materiais, culturais e socioeconômicos para participar de atividades extraescolares. Cabe enfatizar que a mera presença de recursos e materiais não é condição suficiente para determinar o desempenho escolar (Caetano, 2003, Engleend, Luckner, 2004, Ferreira e Marturano, 2002). No entanto, considerando a análise conjunta entre os recursos materiais e humanos, bem como a participação familiar, nesta pesquisa, no grupo de alunos com desempenho insatisfatório, há menor supervisão dos pais tanto em atividades escolares quanto na participação de reuniões escolares. No cotidiano da família, há também menor exigência em relação ao cumprimento de horários para fazer a lição de casa, assistir televisão e para brincar e os membros costumam estar reunidos apenas nos finais de semana, o que implica em menores oportunidades de interação entre pais e filhos durante o decorrer da semana.

Em acréscimo, é possível inferir que tanto as interações dos pais com os filhos quanto à dinâmica familiar podem ser fonte de recursos para que a criança tenha um melhor aproveitamento escolar e apresente desenvolvimento sadio, aprendendo a lidar com suas dificuldades, assim como pode levar a reações de inadaptação e influenciar seu desempenho escolar negativamente. Também se considera que as condições de vida e os recursos do ambiente, associadas ao tempo de dedicação da família no acompanhamento escolar, bem como a qualidade de interação entre pais e filhos, constituem fatores que influenciam o desempenho escolar.

Essa pesquisa realizou uma análise direcionada a um grupo específico e os dados se limitam a um número de participantes, não podendo ser generalizado a uma população escolar maior. Esperase que os dados contribuam para novas pesquisas que busquem delinear, dada a importância atribuída à família, elementos sobre a relação entre desempenho escolar, desenvolvimento intelectual 
e comportamental das crianças. Cabe destacar que há poucas pesquisas, principalmente na literatura nacional, que se dediquem a investigar especificamente a relação entre os recursos humanos, culturais e/ou materiais no ambiente familiar e o desempenho escolar.

Acredita-se que esse trabalho contribui para o entendimento de que a família precisa adquirir como uma atividade da rotina doméstica, independente das condições financeiras, o acompanhamento do processo de aprendizagem da criança, seja na verificação do material escolar, no auxílio à realização das atividades escolares, no estudo para as provas e na participação as reuniões escolares. Também destaca-se a importância da interação familiar, refletida nas possibilidades de compartilhar o diálogo, de fazer uma atividade doméstica em parceria ou mesmo de estar reunida em um momento do dia. Como dito anteriormente, parte-se do pressuposto que a aprendizagem é uma responsabilidade compartilhada entre escola e família.

As limitações desta pesquisa centram-se primeiramente em relação a não ter avaliado indicadores socioeconômicos e a escolaridade os pais dos estudantes investigados. Também considera-se que se a amostra fosse maior, poderíamos ter uma melhor dimensão a respeito da influência familiar em contextos próximos. Uma segunda limitação diz respeito ao espaço geográfico uma vez que a pesquisa foi realizada uma única escola da região metropolitana. Sugere-se que outras pesquisas possam ampliar as regiões para coleta de dados, verificando se há diferenças na participação e organização familiar em virtude das configurações regionais e territoriais.

Não pretendemos esgotar o assunto, mas apontar que essa temática é relevante para o enfrentamento das dificuldades encontradas no processo de aprendizagem e que implicam diretamente no desempenho dos alunos. Mudanças sociais, culturais, políticas e econômicas acabam interferindo na organização e dinâmica familiar, de forma que a família pode transferir para a escola, uma tarefa que também é sua: acompanhar o desempenho de seus filhos. Não estamos afirmando que as famílias fazem isso, no entanto afirmamos que cabe à escola a função de ensinar, mas às famílias cabe tentar, na medida de sua realidade, efetivamente participar do processo de aprendizagem de seus filhos. Outra questão que surge como provocativa para futuras reflexões é como poderíamos contribuir para que a escola conseguisse influenciar as famílias, no sentido de se tornarem atuantes no acompanhamento e na valorização da educação de seus filhos e na participação escolar.

Consideramos que são necessárias novas pesquisas que procurem analisar, numa mesma realidade cultural e social, como essas diferenças podem afetar as famílias, e como podemos contribuir para que essas consigam ajudar seus filhos a conseguir progressos em seu desempenho escolar.

\section{REFERÊNCIAS}

AGUENA, Elaine Cristina. As crenças e as atitudes parentais e desempenho escolar de alunos do ensino fundamental. 2010. 95 f. Dissertação (Mestrado em Educação) - Faculdade de Educação, Universidade Estadual de Campinas, Campinas, SP, 2010. Disponível em: 〈http://goo.gl/y5a9si >. Acesso em: 30 jun. 2015.

ANDRADE, Susanne Anjos, SANTOS, Darci Neves; BASTOS, Ana Cecília et al. Ambiente familiar e desenvolvimento cognitivo infantil: uma abordagem epidemiológica. Revista de Saúde Pública, São Paulo, SP, v. 39, n. 04, p. 606-611. 2005. Disponível em: 〈http://goo.gl/mkUJSz〉. Acesso em: 30 jun. 2015. ISSN 1518-8787. 
BRADLEY, Robert; CORWYN, Robert Flynn. Socioeconomic status and child development. Annual Review of Psychology, v. 53, n. 01, p. 371-399. fev. 2002. Disponível em: 〈http://goo.gl/K59tXB >. Acesso em: 30 jun. 2015. ISSN 0066-4308.

BARBOSA, Maria Ligia de Oliveira. Desigualdade e desempenho: uma introdução à sociologia da escola brasileira. Belo Horizonte: Fino Traço, 2011.

CAETANO, Luciana Maria. Sobre a relação escola e família. In: ASSIS, Mantovani Camargo Mucio; ASSIS, Orly. Zucatto Mantovani de. ENCONTRO NACIONAL DE PROFESSORES DO PROEPRE, 19, 2003, Campinas, SP. Anais eletrônicos... Campinas, 2003.

CARVALHO, Maria Eulina Pessoa de. Modos de educação, gênero e relações escola-família. Cadernos de Pesquisa, São Paulo, SP, v. 34, n. 121, p. 41-58. jan./abr. 2004. Disponível em: <http://goo.gl/6MSINv>. Acesso em: 30 jun. 2015. ISSN 1980-5314.

CARVALHO, Marilia Pinto. Quem são os meninos que fracassam na escola? Cadernos de Pesquisa, São Paulo, SP, v. 34, n. 121, p. 11-40. jan./abr. 2004. Disponível em: <http://goo.gl/WZ8jFj?>. Acesso em: 9 set. 2012. ISSN 1980-5314.

COOPER, Harris, LINDSAY, James, NYE, Barbara. Homework in the home: how student, family, and parenting-style differences relate to the homework process. Contemporary Educational Psychology, v. 25, n. 04, p. 464-487. 1999. Disponível em: 〈http://goo.gl/IgO1ZU〉. Acesso em: 30 jun. 2015. ISSN 0361-476X.

D'AVILA-BACARJI, Keiko Maly Garcia; MARTURANO, Edna Maria; ELIAS, Luciana Carla dos Santos. Recursos e adversidades no ambiente familiar de crianças com desempenho escolar pobre. Paidéia, Ribeirão Preto, SP, v. 15, n. 30, p. 43-55. jan./abr. 2005. Disponível em: 〈http://goo.gl/W23Q4W>. Acesso em: 30 jun. 2015. ISSN 1982-4327.

D’ÁVILA-BACARJI, Keiko Maly Garcia; MARTURANO, Edna Maria; ELIAS, Luciana Carla dos Santos. Suporte parental: um estudo sobre crianças com queixas escolares. Psicologia em Estudo, Maringá, PR, v. 10, n. 01, p. 107-115. jan./abr.2005. Disponível em: 〈http://goo.gl/pdiIoW〉. Acesso em: 30 jun. 2015. ISSN 1807-0329.

DESSEN, Maria Auxiliadora. Contribuições da família para o desenvolvimento da pessoa com deficiência. In: CONGRESSO BRASILEIRO DE PSICOLOGIA DO DESENVOLVIMENTO, 8., 2011, Brasília, DF. Anais eletrônicos... Brasília: ABPD, 2011, p. 724-725.

DESSEN, Maria Auxiliadora; BRAZ, Marcela Pereira. As relações maritais e sua influência nas relações parentais: implicações para o desenvolvimento da criança. In: DESSEN, Maria Auxiliadora; COSTA JUNIOR, Aderson Luis (Org.). A ciência do desenvolvimento humano: tendências atuais e perspectivas futuras. Porto Alegre: Artmed, 2005. p. 132-151.

ENGLUND, Michelle M.; LUCKNER, Amy E. Children's achievement in early elementary school: longitudinal effects of parental involvement, expectation, and quality of assistance. Journal of Educational Psychology, n. 96, n. 04, p. 723-730. 2004. Disponível em: 〈http://goo.gl/aQoUFb〉. Acesso em: 30 jun. 2015. ISSN 1939-2176. 
FERREIRA, Marlene de Cassia Trivellato; MARTURANO, Edna Maria. Ambiente familiar e os problemas de comportamento apresentados por crianças com baixo desempenho escolar. Psicologia: Reflexão e Crítica, Porto Alegre, RS, v. 15, n. 01, p. 35-44. 2002. Disponível em: <http://goo.gl/WZVOOD>. Acesso em: 30 jun. 2015. ISSN 1678-7153.

FERREIRA, Marlene de Cassia Trivellato. As tarefas de desenvolvimento da meninice e a transição para o ensino fundamental. 2005. $197 \mathrm{f}$. Tese (Doutorado em Psicologia) - Faculdade de Filosofia, Ciências e Letras, Universidade de São Paulo, Ribeirão Preto, SP, 2005. Disponível em: < http://goo.gl/xG115G >. Acesso em: 30 jun. 2015.

GUIDETTI, Andreia Arruda. Ambiente familiar e desempenho acadêmico de crianças do ensino fundamental. 2007. 99 f. Dissertação (Mestrado em Educação) - Faculdade de Educação, Universidade Estadual de Campinas, Campinas, SP, 2007. Disponível em: 〈http://goo.gl/Aqs37E〉. Acesso em: 30 jun. 2015.

GUIDETTI, Andreia Arruda; MARTINELLI, Selma de Cassia. Desempenho em leitura e suas relações com o contexto familiar. In: SANTOS, Acácia Aparecida Angeli; BORUCHOVITCH, Evely; OLIVEIRA, Kátia Luciane de. Cloze: um instrumento de diagnóstico e intervenção. São Paulo: Casa do Psicólogo, 2009.

GUIDETTI, Andreia Arruda; MARTINELLI, Selma de Cassia. Questionário sobre os suportes e recursos do ambiente familiar. (Mimeografado). Campinas: FE: UNICAMP, 2005.

JIANG, Yimin. Family environment and academic achievement in Nanjing secondary schools (China). Dissertation Abstracts International Section A - Humanities and Social Sciences, v. 64, p. 4-12. 2004.

MADAUS, George; AIRASIAN, Peter; KELLAGHAN, Thomas. Insumos escolares, processos e recursos. In: BROKE, Nigel; SOARES, J. F. Pesquisa em eficácia escolar. Belo Horizonte, Editora UFMG, 2008.

MARCHESI, Alvaro. O que será de nós, os maus alunos? Porto Alegre: Artmed, 2006.

MARTURANO, Edna Maria. O inventário de recursos do ambiente familiar. Psicologia: Reflexão e Crítica, Brasília, DF, v. 19, n. 03, p. 498-506. 2006. Disponível em: 〈http://goo.gl/XghXmD〉. Acesso em: 30 jun. 2015. ISSN 1678-7153.

MARTURANO, Edna Maria. Recursos no ambiente familiar e dificuldades de aprendizagem na escola. Psicologia: Teoria e Pesquisa, Brasília, DF, v. 15, n. 02, p. 135-142. mai./ago. 1999. Disponível em: <http://goo.gl/pF9sfG >. Acesso em: 30 jun. 2015. ISSN 1806-3446.

MIDGETT, Jonathan; BELSITO, Laura, RYAN, Bruce A.; ADAMS, Gerald. Children's and parent's perceptions of parental attitudes and behaviors pertaining to academic achievement. In: ODDONE, P.; ELIZABETH, E.; VIOLAT, C. The changing and child development. Andershot, England: Ashagate Publishing, 2000. p. 162-175.

MILLER, Alan. The effects of beliefs perceptions and values on parent's expectations for their childrens' academic achievement. Dissertation Abstracts International Section A - Humanities and Social Siences. 1999. 
MOREIRA, Rafaela Marcolino; PAES, Tais Galdioli; PEDROZA, Regina Lucia Sucupira. O adolescente e sua relação familiar: concepções de adolescentes de Ensino Médio. In: CONGRESSO BRASILEIRO DE PSICOLOGIA DO DESENVOLVIMENTO, 8., 2011, Brasília, DF. Anais eletrônicos... Brasília: ABPD, 2011. p. 847-849.

OSTI, Andreia. Representações de alunos e professores sobre ensino e aprendizagem. 2010. 189 f. Tese (Doutorado em Educação) - Faculdade de Educação, Universidade Estadual de Campinas, Campinas, SP, 2010. Disponível em: 〈http://goo.gl/HJb2vF>. Acesso em: 30 jun. 2015.

OSTI, Andreia; BRENELLI, Rosely Palermo. Representações de alunos (com e sem dificuldades de aprendizagem) sobre suas experiências de aprendizagem e ambientes significativos. Schème Revista Eletrônica de Psicologia e Epistemologia Genéticas, Marília, SP, v. 02, n. 04, p. 35-64. ago./dez. 2009. Disponível em: 〈https://goo.gl/fwun8f>. Acesso em: 30 jun. 2015. ISSN 1984-1655.

OSTI, Andreia; MARTINELLI, Selma de Cássia. Desempenho escolar: análise comparativa em função do sexo e percepção dos estudantes. Educação e Pesquisa, São Paulo, SP, v. 40, n. 01, p. 4959. 2014. Disponível em: 〈http://goo.gl/vcqONd>. Acesso em: 30 jun. 2015. ISSN 1678-4634.

PATRINOS, Harry. Estimating the returns to education: accounting for heterogeneity in ability. In: INTERNATIONAL CONFERENCE ON ECONOMICS OF EDUCATION. Anais eletrônicos... Dijon, 2006.

POLONIA, Ana da Costa, DESSEN, Maria Auxiliadora. Em busca de uma compreensão das relações entre família e escola. Psicologia Escolar e Educacional, Campinas, SP, v. 09, n. 02, p. 303-312. 2005. Disponível em: 〈http://goo.gl/vnI6HY>. Acesso em: 30 jun. 2015. ISSN 1413-8557.

RANDALL, Laura. Factors affeting learning and cost effective schooling in Latin America, Argentina, Brazil, Chile and Mexico.Lewiston. Nova York: Edwin Mellen Press, 2005.

SANTOS, Acacia Aparecida Angeli; MARTINELLI, Selma de Cássia; MONTEIRO, Rebeca Magalhães. Suportes e recursos familiares: relações com o contexto escolar. In: BAPTISTA, Makilim Nunes; TEODORO, Maycoln (Org.). Psicologia de família: teoria, avaliação e intervenção. Porto Alegre: Artmed, 2012.

SANTOS, Acacia Aparecida Angeli; RUEDA, Fabian Javier; BARTHOLOMEU, Daniel. Avaliação dos aspectos afetivos envolvidos nas dificuldades de aprendizagem. In: MARTINELLI, Selma de Cassia; SISTO, Fermino Fernandes. Afetividade e dificuldades de aprendizagem: uma abordagem psicopedagógica. São Paulo: Vetor, 2006.

SANTOS, Luciana Carla dos; MARTURANO, Edna Maria. Crianças com dificuldade de aprendizagem: um estudo de seguimento. Psicologia: Reflexão e Critica, Porto Alegre, RS, v. 12, n. 02, p. 377-394. 1999. Disponível em: 〈http://goo.gl/LyQVq9>. Acesso em: 30 jun. 2015. ISSN 1678-7153.

SILVA, Daiana Cristina; VARANI, Adriana. A relação família e escola: implicações no desempenho escolar dos alunos dos anos iniciais do ensino fundamental. In: CONGRESSO INTERNACIONAL GALEGO-PORTUGUÊS DE PSICOPEDAGOGIA, 10., 2009, Braga, Portugal. Anais eletrônicos... Braga: Universidade do Minho, 2009. p. 1714-1728. 
SPERA, Christopher. Adolescent's perceptions of parental goals, practices and styles in relation to their motivation and achievement. The Journal of Early Adolescence, v. 26, p. 456-490. nov. 2006. Disponível em: <http://goo.gl/hknUq5>. Acesso em: 30 jun. 2015. ISSN 1552-5449.

STEENSEL, Roel Van. Relations between socio-cultural factors, the home literacy environment and children's literacy development in the first years of primary educations. Journal of Research in Reading, v. 29, n. 04, p. 367-382. nov. 2006. Disponível em: 〈http://goo.gl/3ku2Gf〉. Acesso em: 30 jun. 2015. ISSN 1467-9817.

SWARTZ, Meredith. The academic achievement of mexican-american students: associations with family environment and parental level of acculturation. Dissertation Abstracts International Section B: The Sciences and Engineering. 64 (5-B), 2003.

\section{Como citar este documento:}

OSTI, Andreia. Contexto familiar e o desempenho de estudantes de uma escola no interior de São Paulo. ETD Educação Temática Digital, Campinas, SP, v. 18, n. 2, abr./jun. 2016. ISSN 1676-2592. Disponível em: <http://periodicos.sbu.unicamp.br/ojs/index.php/etd/article/view/8637508>. Acesso em: 04 jul. 2016. doi:http://dx.doi.org/10.20396/etd.v18i2.8637508. 